Пермский государственный медицинский университет имени академика Е.А. Вагнера (Пермь, Россия)
ОТДЕЛЬНЫЕ ДЕМОГРАФИЧЕСКИЕ ПОКАЗАТЕЛИ, ФАКТОРЫ РИСКА И СОВРЕМЕННЫЕ НАПРАВЛЕНИЯ В СКРИНИНГЕ ЗЛОКАЧЕСТВЕННЫХ НОВООБРАЗОВАНИЙ (ОБЗОР ЛИТЕРАТУРЫ)

А.Н. Жигулев, В.Ю. Мишланов, О.А. Орлов, А.В. Каткова

\section{SPECIFIC DEMOGRAPHIC DETERMINANTS, THREATS, AND MODERN TRENDS IN MALIGNANT NEOPLASM SCREENING (REVIEW)}

А.Н. Жигулев Аспирант кафедры пропедевтики внутренних болезней №1,

Пермский государственный медицинский университет имени академика Е.А. Вагнера, 614000, Россия, Пермь, Петропавловская улица, 26. Тел.: 8 (342) 217-10-31.

В.Ю. Мишланов Доктор медицинских наук, профессор, член-корреспондент РАН, заведующий кафедрой пропедевтики внутренних болезней №1.

О.А. Орлов

Доктормедицинских наук, профессор, заведующий кафедрой онкологии, лучевой терапии и лучевой диагностики.

A.В. Каткова

Кандидат медицинских наук, доцент кафедры пропедевтики внутренних болезней №1.

A.N. Zhigulev Postgraduate student, Department of Propaedeutics of Internal Diseases No. 1, Wagner Perm State Medical University, 614000, Russia, Perm, Petropavlovskaya str., 26. Phone: 8 (342) 217-10-31.

V.Yu. Mishlanov Doctor of Medicine, Professor, Corresponding Member of the Russian Academy of Sciences, Head of Department of Propaedeutics of Internal Diseases No. 1.

O.A. Orlov

Doctor of Medicine, Professor,

Head of the Department of Oncology, Radiation Therapy and Radiation Diagnostics.

A.V. Katkova Candidate of Medicine, Associate Professor of Propaedeutics of Internal Diseases No. 1.

В данной работе была поставлена задача осветить отдельные демографические показатели, связанные со злокачественными новообразованиями в мире заболеваемость и смертность, а также их динамику. Представлена информация по экономическому ущербу и глобальному бремени, связанному с онкологическими заболеваниями. Важной частью обзора является представление основных факторов риска, оказывающих влияние на развитие злокачественных новооб- 
разований (3НО). Также представлен обзор мирового опыта по проведению, изучению и оценке эффективности методов скрининга отдельных злокачественных опухолей.

Ключевые слова: злокачественные новообразования, рак, демография, факторы риска, скрининг.

The paper covers specific demographic determinants related to malignant neoplasms throughout the world including morbidity, mortality, and their changes in time. The paper presents data on economic damage and global burden of disease related to oncological diseases. The important part of the review includes the presentation of main threats that influence the development of malignant neoplasms (MN). The paper also gives a review of international practices aimed at implementation, analysis, and efficiency estimation of screening methods for particular types of malignant neoplasms.

Keywords: malignant neoplasms, cancer, demography, threats, screening.

K аждый год 7 миллионов жизней можно было бы спасти при проведении профилактики и лечении предопухолевых заболеваний. [1]. Показатели заболеваемости ЗНО могут существенно возрасти в будущем, до 15 миллионов новых случаев в 2020 году, большинство из которых будет зарегистрировано в развивающихся странах [2].

Рост заболеваемости основными неинфекционными заболеваниями, такими как гипертоническая болезнь, атеросклероз, психическая патология, сахарный диабет и опухолевые болезни, обусловлен рядом причин. Среди них можно назвать ускорение темпов жизни, технический прогресс, использование новых синтетических материалов в промышленности, строительстве, быту, ухудшение экологических условий жизнедеятельности человека, а также изменение качества питания. Рост заболеваемости и увеличение смертности от $3 \mathrm{HO}$ наносят обществу огромный социальный и экономический ущерб, связанный с глубокой инвалидизацией заболевших и ранней смертностью. Среди населения развитых стран $3 \mathrm{HO}$ являются причиной смертности каждого пятого из умерших [3, 4]. Темпы роста заболеваемости $3 \mathrm{HO} \mathrm{в}$ последние годы занимают второе место, уступая лишь сердечно-сосудистой патологии [5]. Возраст - важный фактор в развитии онкологических заболеваний. Многие злокачественные опухоли, в том числе рак предстательной железы, желудка и толстой кишки, чаще всего встречаются у людей в возрасте после 60 лет. Более $60 \%$ 3НО выявляются после 65 лет. После достижения человеком 25-летнего возраста вероятность возникновения таких заболеваний удваивается каждые пять лет. Рост числа ЗНО при увеличении средней продолжительности жизни, по-видимому, является результатом сочетания более сильного и длительного воздействия канцерогенных веществ и изменения отдельных показателей иммунной системы организма (иммуносупрессия или иммунопотенция). Россия, по данным ВОЗ, входит в тройку европейских стран с наивысшими показателями смертности от 3НО [6]. В отдельных регионах, где преобладают старшие возрастные группы, в популяции чаще встречаются 3НО, большая часть из которых имеет неблагоприятный прогноз в плане раннего выявления и радикального лечения. Для таких территорий высока значимость и целесообразность внедрения современных методов раннего выявления онкологических больных, приоритетное финансирование онкологической службы, и мероприятий, направленных на диспансеризацию определенных групп населения.

Наиболее важным показателем бремени для любой локализации рака, за исключением немеланомного рака кожи, является стандартизованный коэффициент смертности, который рассчитывается на население и выражает вероятность развития или смерти от рака для среднестатистического индивида [7]. Важным аспектом уровня заболеваемости и смертности от 3НО также является качество статистики и регистрационных систем в странах с низким и средним уровнем доходов [8], к которым можно отнести и Россию. Кроме того, ограничивают достоверность данных о смертности поздняя диагностика, ошибки, недостаточная подготовка специалистов, участвующих в сборе информации и нехватка ресурсов $[9,10]$.

3НО причиняют наибольший экономический ущерб, возглавляя список самых затратных болезней, в связи с колоссальными расходами на финансирование лечения и выплаты по нетрудоспособности. По данным Американского национального института здоровья в 2007 году онкологические заболевания нанесли экономике США ущерб в размере 219,2 млрд долларов, из них 89 млрд - это прямые расходы, 18,2 млрд - финансовые потери при снижении производительности и 112 млрд - ущерб от смерти заболевших [6]. В Российской Федерации экономический ущерб может достигать 200 млрд рублей ежегодно [11]. Последние описательные исследования отмечают увеличение глобального бремени злокачественных опухолей, в первую очередь касающиеся числа случаев заболевания и смерти $[12,13]$. Количество случаев заболевания и смерти чувствительны к возрастной структуре. В странах с высоким уровнем дохода выше доля населения старших возрастных групп и, соответственно, выше уровень заболеваемости ЗНО [14].

\section{Отдельные демографические показатели}

База данных о смертности ВОЗ [15] содержит данные по возрасту, полу и причине смерти на основе регистрации актов гражданского состояния, а также других источников: переписей населения, демографических обследований и регистров населения. В базу данных включаются страны с населением более 
2 миллионов жителей, осуществляющие регистрацию причин смерти в соответствии с Международной классификацией болезней (МКБ) 9-й или 10-й редакции [16]. Все случаи смерти, прошедшие регистрацию по МКБ-9, были преобразованы в МКБ-10 [17]. Охват регистрацией актов гражданского состояния о причинах смерти был практически 100\% в Европе, Северной Америке, Океании, Японии и Корее. Некоторые страны Латинской Америки, бывшего СССР, других частей Азии, Южной Африки, не предоставляют данные о смертности в ВО3, поскольку не используют систему кодирования МКБ или имеют неполные регистрационные данные. Семь стран (Армения, Кипр, Сальвадор, Египет, Гватемала, Грузия, и Турция) были исключены, потому что предварительный анализ показал необъяснимо низкий уровень смертности от рака. Остальные 60 стран с населением 2,006 млрд жителей в 2010 году были распределены по семи регионам: Северная Америка, Латинская Америка, Европа (кроме стран бывшего Советского Союза), страны бывшего Советского Союза, Япония / Корея, другие страны Азии / Южной Африки и Океании. Япония и Корея были классифицированы отдельно от континентальной Азии из-за высокой заболеваемости раком желудка. Из африканского континента в анализ была включена только одна страна - ЮАР. Показатели смертности были стандартизированные по возрасту на 100000 человеко-лет для всех возрастов прямым методом на основе мирового стандарта населения [18]. В общую статистику смертности от онкологических заболеваний - злокачественные и доброкачественные новообразования (C00-D48) были объединены для повышения достоверности и сопоставимости данных между странами и включают смертельные доброкачественные новообразования. Смертность была рассчитана для наиболее распространенных локализаций рака в мире по МКБ-10: желудок (С16), прямая кишка (C18-21), печень (С22), легкое (С33-34), у женщин - молочные железы (С50), матка (С53 и С54), у мужчин - предстательная железа (C61) [17]. Показатели рака тела и шейки матки были объединены [19, 20]. В 2010 году в 60 странах зарегистрировано свыше 3,7 млн случаев смерти от рака. В 2000 году эта цифра составляла 3,2 млн случаев.

С 2000 по 2010 год отмечалось некоторое снижение смертности от рака всех локализаций ежегодно на $1,2 \%$ у мужчин и $0,8 \%$ у женщин. В большинстве стран (41/60 для мужчин и для женщин 35/60, включенных BO3), это снижение 1\% в год или выше. Та же тенденция была отмечена по результату совместного проекта Международного агентства исследования рака с ВО3 (International Agency for Research on Cancer - IARC) GLOBOCAN 2002 года [21] и 2012 года [22]. И только смертность от рака легкого среди женщин увеличилась в большинстве стран. Смертность от рака легких, желудка и печени напрямую связана с факторами риска. При этом тенденции смертности коррелиру- ют с показателями заболеваемости, аналогичными в большинстве стран, менее подверженных влиянию экономического неравенства. Сближение доли курящих между мужчинами и женщинами отражается на увеличении уженщин заболеваемости раком легких и, как следствие, увеличение у них смертности от данной патологии. Хотя в целом курение табака снизилось, смертность от рака легких у мужчин и женщин приближается к равному показателю в нескольких европейских странах и ожидается, что будет продолжаться рост у женщин во всем мире [17].

Наибольший вклад в структуру снижения смертности внесли мероприятия по борьбе со злокачественными новообразованиями желудка (2,7\% у мужчин и 2,8\% у женщин). За последние 10 лет значительное снижение произошло в Континентальной Азии, Латинской Америке и странах бывшего Советского Союза. Снижение заболеваемости раком желудка в последние несколько десятилетий объясняют диагностикой Helicobacter Pylori, контролем и лечением $[23,24]$. Менее ста лет назад рак желудка был наиболее распространенной формой рака в Соединенных Штатах и, возможно, во всем мире. Несмотря на то, что рак желудка больше не является самым распространенным видом рака, он остается второй ведущей причиной смертности от ЗНО в мире, и наиболее распространенной формой рака в Восточной Азии [25].

Среди всех видов ЗНО рак желудка является одной из ведущих причин смерти в последние десятилетия, и количество жертв от этой формы рака постоянно растет в Азии и по всему земному шару [26]. Способствуют увеличению смертельных случаев от рака желудка экологические факторы, ожирение и гастроэзофагеальная рефлюксная болезнь [27]. При постановке первичного диагноза в 23\% случаев $3 \mathrm{HO}$ желудка локализованы, в 32\% обнаруживаются метастазы в лимфатических узлах, а в 34\% - является метастатическим [28]. Рак желудка занимает четвертое место по распространенности в мире. Это заболевание гораздо чаще встречается в Японии, Центральной Европе, Скандинавии, Гонконге, Южной и Центральной Америке, России и бывших странах СССР, Китае и Корее. Рак желудка - одна из основных причин смертности в мире. Основной гистологический тип рака желудка аденокарцинома (95\%). Оставшиеся 5\% - лимфомы, саркомы, карциноидные опухоли и другие редкие виды опухолей. В последнее десятилетие появляется все большее число исследований, сосредоточенных на общей выживаемости больных с этой патологией. Результаты этих исследований указывают на то, что показатели выживаемости в развивающихся странах ниже, чем в развитых [29].

Показатели заболеваемости раком желудка значительно колеблются среди мужчин и женщин и в разных странах. Показатели заболеваемости в 2-3 раза выше у мужчин, чем уженщин [30]. Самые высокие показатели заболеваемости наблюдаются в Восточной 
Азии, Восточной Европе и Южной Америке, в то время как самые низкие показатели наблюдаются в Северной Америке и большей части Африки. Например, ежегодные темпы прироста заболеваемости раком желудка, стандартизированные по возрасту на 100000 мужчин, составляют 65,9 в Корее против 3,3 в Египте [31]. В Соединенных Штатах заболеваемость относительно низка, особенно среди белого населения. Расчетная заболеваемость на 100000 составляют 7,8 и 3,5 у неиспаноговорящих белых мужчин и женщин, соответственно. Показатели также отличаются по расам. В Соединенных Штатах они выше у латиноамериканцев (13,9 на 100000 у мужчин и 8,2 на 100000 женщин), чем среди нелатиноамериканского белого населения [32]. У коренных народов (в частности инуиты) в приполярной области и Маори в Новой Зеландии встречаемость 3 НО выше [33]. Существует доказательство того, что инфекция желудка Helicobacter pylori тесно связана с началом развития рака и лимфомы желудка. [34-36] Международное агентство по изучению рака (IARC) классифицирует инфекции H. Pylori в качестве причины некардиальной карциномы желудка и высокодифференцированной $\beta$-клеточных лимфомы лимфоидной ткани слизистой оболочки желудка [37, 38]. По сравнению с населением в целом, люди с язвенной болезнью двенадцатиперстной кишки имеют меньшую вероятность развития рака желудка [39].

Смертность от колоректального рака, рака молочной железы и рака матки имеет тенденцию к снижению в странах с высоким уровнем дохода из-за скрининга и лечения, хотя остается высокой. Смертность от рака молочной железы и колоректального рака возросла в странах с низким и средним уровнем дохода за счет увеличения частоты встречаемости факторов риска - ожирение, отсутствие физической активности и изменения, происходящие в репродуктивной сфере [40]. Это наблюдается при раке молочной железы в Континентальной Азии и Южной Африке и при колоректальном раке в Латинской Америке и Азии. Наибольшее снижение заболеваемости ЗНО матки, чаще шейки матки, наблюдается в Латинской Америке, Азии и бывшем Советском Союзе. Это снижение связано с увеличением частоты использования скрининговых методов диагностики заболеваний шейки матки. Ожидается дальнейшее снижение, учитывая актуальность, эффективность и надежность этих методик, а также проводимой вакцинации против вируса папилломы человека [41].

Общий уровень заболеваемости у мужчин (205 на 100 000) почти на 25\% выше, чем у женщин (165 на 100 000). Уровень заболеваемости среди мужчин различается почти в пять раз по различным регионам мира. С 79 на 100000 в Западной Африке до 365 на 100000 в Австралии / Новой Зеландии. Значительно увеличивает показатель общей заболеваемости $3 \mathrm{HO}$ в данных странах очень высокий уровень заболеваемости раком простаты. В меньшей степени варьи- руются показатели заболеваемости среди женщин, в пределах от 103 на 100000 в Южно-Центральной Азии до 295 на 100000 в Северной Америке. С точки зрения смертности, кумулятивный риск смерти от рака на 20\% выше в более развитых, чем менее развитых регионах у мужчин, и на 10\% выше у женщин. Риск смерти от рака среди мужчин самый высокий в Центральной и Восточной Европе (19\%) и самый низкий в Западной Африке (7\%). В противоположность этому, самый высокий риск смерти от рака у женщин в Малайзии и Восточной Африке (12\% для каждого региона), а самый низкий в Восточной Азии (8\%) и Южно-Центральной Азии (7\%).

Во всем мире бремя болезни продолжает увеличиваться в основном из-за старения населения и распространения деятельности, связанной с производством канцерогенов [42]. Новые данные и прогнозы глобального бремени рака, представленные в Мировом Докладе по Раку 2014 года [43] свидетельствуют о том, что заболеваемость раком увеличится. Ожидается увеличение числа новых случаев рака до 25 миллионов в течение следующих двух десятилетий [44]. В целом можно отметить, что показатели смертности от рака легких, молочной железы, простаты и колоректального рака остаются высокими в странах с низким или средним уровнем доходов. Обратная ситуация наблюдается в странах с высоким уровнем дохода за счет изменения воздействия факторов риска. В должной мере на борьбу с опухолевыми заболеваниями в этих странах отдан приоритет сил и средств, направленных на уменьшение бремени смертности. Многие современные исследования подчеркивают возрастающую необходимость превентивных мер, направленных на сокращение факторов риска, профилактику и раннюю диагностику онкологических заболеваний.

\section{Факторы риска возникновения $3 \mathrm{HO}$}

Заболеваемость раком желудка в Соединенных Штатах в четыре раза сократилась с 1930 года до примерно семи случаев на 100000 человек [45]. Причины этого поразительного снижения заболеваемости до конца не изучены, но предполагается их связь с улучшенными условиями хранения пищевых продуктов и изменениями в рационе питания, такими, как снижение потребления соли. Некоторые группы американцев подвергаются повышенному риску заболеть. Это пожилые пациенты с атрофическим гастритом или анемией, пациенты со спорадической аденомой желудка [46], семейным аденоматозным полипозом [47], иммигранты в этнических группах из стран с высокими показателями заболеваемости раком желудка, а также больные неполипозным наследственным раком толстой кишки [48-50].

Генетические и экологические факторы включают семейную историю рака желудка; низкое потребление фруктов и овощей; потребление соленой, 
копченой, или неправильно хранившейся пищи; курение [51, 52]. Значительная часть страданий и смерти от рака могли быть предотвращены путем более систематических усилий по сокращению употребления табака, улучшению питания и усилению физической активности, снижению веса, а также более широкому использованию разработанных скрининговых тестов. Отказ от курения и возможно профилактика пассивного курения, поддержание оптимального веса, физическая активность на протяжении всей жизни и следование здоровой диете могут в значительной степени уменьшить риск развития рака или смерти от него $[53,54]$. Американское онкологическое общество (ACS) оценивает, что в 2015 году около 171000 случаев смерти были вызваны только курением [55]. Кроме того, от одной четверти до одной трети из 1658370 случаев заболеваний раком в 2015 году может быть связано с плохим питанием, отсутствием физической активности, избыточным весом и ожирением [53-55].

В 2014 году отмечался 50-летний юбилей доклада департамента США по здравоохранению, посвященному вопросу курения и здоровья. В этом знаковом отчете постулировалось, что курение сигарет вызывает рак легких [56], и с тех пор другие табачные изделия, в том числе сигары и бездымный табак, были также причинно связаны с раком. Значительные успехи в области борьбы с курением были сделаны после доклада. Тем не менее, с 1964 года зарегистрировано более 20 миллионов случаев смерти от курения. Употребление табака остается единственной значительной предотвратимой причиной заболеваний и преждевременной смерти [57]. Эта привычка повышает риск развития рака легкого, полости рта, гортани, глотки, пищевода, желудка, кишечника и толстой кишки, печени, поджелудочной железы, почек, мочевого пузыря, шейки матки и яичников (мукозный тип), а также миелоидной лейкемии $[57,58]$. Ограниченные, но постоянно растущие данные свидетельствуют о том, что длительное, интенсивное курение увеличивает риск развития рака молочной железы, особенно среди женщин, которые начали курить до родов первого ребенка $[59,60]$. Воздействие от пассивного курения также увеличивает риск развития рака легких у взрослых [57, 61].

Избыточный вес и ожирение связаны с повышенным риском развития многих видов рака, включая рак молочной железы у женщин в постменопаузе [62], толстой и прямой кишки [63], эндометрия, почек и поджелудочной железы [63, 64], а также аденокарциномы пищевода [65]. Кроме того, ожирение, вероятно, повышает риск развития рака желчного пузыря [63,], а также может увеличить риск возникновения рака печени, шейки матки и яичников, множественной миеломы, неходжкинской лимфомы и агрессивных форм рака простаты [66-68]. Хотя сведения о взаимосвязи между потерей веса и риском рака ограничены, иссле- дования показывают, что потеря веса может снизить риск рака молочной железы в постменопаузе [69-71]. Физическая активность воздействует различными путями, уменьшая риск развития некоторых видов рака, включая рак молочной железы, толстой кишки и эндометрия [72]. Кроме того, регулярная физическая активность помогает поддерживать оптимальный вес тела благодаря увеличению расхода энергии. Более высокий уровень потребления некрахмалистых овощей и фруктов связан с более низким риском рака ротовой полости, глотки, гортани, пищевода, желудка [73]. Последние данные также свидетельствуют, что потребление некрахмалистых овощей снижает риск әстроген рецептор-отрицательных опухолей молочной железы [74]. Частое употребление обработанного или красного мяса доказательно связано с повышенным риском развития колоректального рака [75], но лишь ограниченно и косвенно связано с повышенным риском развития рака поджелудочной железы [76]. Употребление алкоголя является доказанной причиной рака ротовой полости, глотки, гортани, пищевода, печени, толстой и прямой кишки, молочной железы, и есть некоторые доказательства связи с раком поджелудочной железы $[65,72,77]$. В сочетании с употреблением табака, употребление алкоголя повышает риск развития рака ротовой полости, гортани и пищевода гораздо больше, чем отдельное влияние только алкоголя или только табака [72].

Прямые связи между углеводами и раком продолжают демонстрировать смешанные результаты. Проведенные систематические обзоры скорее предполагают, чем подтверждают потенциальные связи между углеводами (или разными типами углеводов) и конкретными видами рака. Примеры таких отношений, указывают на связь между сахарозой и колоректальным раком, лактозой и раком яичников, фруктозой и раком поджелудочной железы, а также колоректальным раком и раком эндометрия. С другой стороны, ряд проведенных мета-исследований не обнаруживает взаимосвязи между углеводами и риском развития рака желудочно-кишечного тракта. Собрано очень мало доказательств, чтобы показать какую-либо связь между углеводами и раком полости рта, глотки, желудка, легких, шейки матки, простаты почек. В тоже время имеются убедительные доказательства объясняющие, почему углеводы могут влиять на неалкогольную жировую болезнь печени, неалкогольный стеатогепатит. Отмечается связь углеводов с развитием метаболического синдрома (например, гиперинсулинемия) с его результирующим воздействием на стимуляцию инсулиноподобных факторов роста 1 , пролиферацию клеток и дерегулирование апоптоза.

\section{Мировой опыт диагностики рака желудка}

Ошибочный диагноз является самым большим препятствием на пути лечения рака желудка [78-80]. В большинстве случаев, когда болезнь обнаружена и 
правильно установлен диагноз - хирургический метод лечения является основным вариантом лечения. Программы раннего выявления и скрининга имеют решающее значение для улучшения прогноза $3 \mathrm{HO}$ желудка [81, 82].

В настоящее время мировое медицинское сообщество не имеет достаточного количества доказательств, связанных с высокой эффективностью скрининга ЗНО желудка. Несколько методов скрининга, в том числе с использованием рентгенофлюорографии желудка с взвесью сульфата бария, эндоскопии желудка и анализа пепсиногена в сыворотке крови, были предложены в качестве методов скрининга для раннего выявления рака желудка. Рандомизированных исследований, оценивающих влияние скрининга на смертность от рака желудка, нет $[83,84]$. Даже в районах повышенной опасности положительная прогностическая ценность скрининг-тестов может быть очень низкой. К примеру, в большой программе скрининга рака желудка в городе Вакаяма (Япония) в которой участвовало 17647 мужчин в возрасте от 40 до 60 лет, проводилось исследование пепсиногена в сыворотке крови и рентгенофлюорография желудка с взвесью сульфата бария. За 7-летний период частота выявленной патологии составила 0,85\%. При этом, частота обнаружения 3НО составила $0,28 \%$. Отдаленные результаты проводимого исследования показали, что за 7-летний период не произошло сокращения смертности от рака желудка в исследуемой группе по сравнению с подобранными по возрасту группами населения, не проходившими скрининг [85].

Национальная программа популяционного скрининга рака желудка с использованием рентгенофлюорографии желудка с взвесью сульфата бария ведется в Японии с 1960-х годов. Отмечено, что снижение смертности от рака желудка в Японии совпадает со снижением смертности в странах, в которых отсутствуют программы скрининга. Исследования в Японии показывают снижение смертности при раке желудка у людей, которые прошли скрининг, но результаты проспективных исследований оказываются противоречивыми. [83, 84]. Экспериментальное исследование проводилось в Коста-Рике с использованием тех же приемов, которые применяются в национальной программе Японии (на основе консультаций с японскими специалистами) [17]. Несмотря на убеждение авторов, что их результаты предоставляют веские доказательства того, что по заведенному порядку скрининг будет снижать смертность от рака желудка, они пришли к выводу, что расходы на осмотр и рентгенофлюорография желудка с взвесью сульфата бария будут слишком высокие по стране. Похожее скрининговое исследование в Венесуәле было начато в 1980 году. Эффективность этой программы по сокращению смертности от рака желудка оценивалась путем исследования случай-контроль, и не показало снижения смертности от данного заболевания.
Эндоскопия является более чувствительным методом выявления рака желудка, чем рентгенологический [86]. Когортное исследование эндоскопического скрининга проводилось в провинции Линьчу (Китай), где показатели заболеваемости раком желудка высоки. В данном исследовании принимало участие 4394 человека взрослого населения в возрасте от 35 до 64 лет. Жители были обследованы в среднем за 4,5 года. При анализе отдаленных результатом отмечено отсутствие влияния эндоскопического скрининга на уровень смертности от рака желудка среди отобранных лиц. Стандартизированный коэффициент смертности (СКС) для рака желудка через 10 лет после начального скрининга составил 1,01 (95\% доверительный интервал, 0,72-1,37). СКС от всех причин смерти оказался среди участников значительно ниже, чем в общей популяции, поскольку люди с гипертонической болезнью, заболеваниями печени и хронической обструктивной болезнью легких не имели права участвовать [87].

Одной из используемых методик в мире по скринингу 3 НО желудка является анализ пепсиногена в сыворотке крови. При этом, нет никаких исследований, оценивающих влияние использования данной методики на смертность от рака желудка, и существуют значительные ограничения на его использование в качестве материала для скринингового теста. Низкий уровень пепсиногена в сыворотке указывает на наличие атрофического гастрита и, следовательно, применимо к обнаружению предположительно прекурсоров для кишечного типа рака желудка, а не диффузного типа. Кроме того, нет стандартных пороговых значений отклонений. Эрадикация H. pylori и применение ингибиторов протонной помпы снижает уровень пепсиногена, что усложняет интерпретацию результатов в условиях широкого применения этого типа исследования. В Японии проводилось измерение в сыворотке крови уровней пепсиногена I и II и обследование пациентов эндоскопическим методом. Эта комбинация обеспечила чувствительность методике 84,6\%, специфичность 73,5\%.

Регулярное применение скрининговых методов диагностики заболеваний шейки матки и кишечника позволит предотвратить развитие рака путем выявления и удаления или лечения предраковых аномалий [88]. Скрининговые тесты могут также улучшить выживаемость и снизить смертность путем выявления рака на ранней стадии, когда лечение более эффективно. Вакцины вируса папилломы человека (ВПЧ) и вируса гепатита B (HBV) предотвращают потенциально канцерогенные инфекции, которые могут привести к развитию рака.

\section{Заключение}

Демографические наблюдения показывают, что частота встречаемости злокачественных новообразований в мире ежегодно будет расти и это будет 
сопряжено с продолжающимся увеличением экономического ущерба от болезни. Актуальным направлением в развитии современной онкологии продолжает оставаться, в первую очередь, ранняя скрининговая диагностика злокачественного процесса. Лишь методы своевременной диагностики позволят проводить радикальное лечение, что несомненно приведет к глобальному снижению преждевременной смертности от рака. К сожалению, на сегодняшний день профессиональное медицинское сообщество не определило единого подхода к ранней диагностике ЗНО даже одной локализации - рака желудка.

\section{Список литературы}

1. Jemal A., Bray F., Center M.M., Ferlay J., Ward E., Forman D. Global cancer statistics. // CA Cancer J Clin. 2011. Vol. 61. - P. 69-90.

2. Pourboseingholi M.A., Vahedi M., Baghestani A.R. Burden of gastrointestinal cancer in Asia; an overview. // Gastroenterol Hepatol Bed Bench. 2015. - Vol. 8. - P. 19-27.

3. Axel E.M., Dvoirin V.V. Statistics of malignant neoplasms (incidence, mortality, trends, socio-economic damage, life expectancy). // Moscow: VONTS AMN SSSR. 1992. - P. 308.

4. Hoffman George. Cancer caused by exposure to low doses: an independent analysis of the problems (translation from English). // Socio-ecological Union. 1994.

5. Yagudina R.I., Kulikov A.Yu., Arinina E.E. Pharmacoeconomics in Oncology. // «Шико». 2011. - P. 10.

6. Abmad O.B., Boschi-Pinto C., Lopez A.D., et al. Age Standardization of Rates: A new WHO Standard. // Geneva: World Health Organization. 2001.

7. Jong-wook L. Global health improvement and WHO: shaping the future. // Lancet. 2003. - Vol. 362(9401). P. 2083-2088.

8. Haghighi M.H., Debghani M., Teshizi S.H., Mabmoodi H. Impact of documentation errors on accuracy of cause of death coding in an educational hospital in Southern Iran. // HIM J. 2014. - Vol. 43(2). - P. 35-42.

9. Silva J.A., Yamaki V.N., Oliveira J.P., et al. Commitment in the completion of the medical death certification. Evaluation in Belem, Para, Brazil in 2010. // Rev Assoc Med Bras. 2013. - Vol. 59(4). - P. 335-340.

10. Chissov V.I., Starinskiy V.V., Aleksandrova L.M., Lutkovskiy A.S., Savinov V.A., Petrova G.V., Gretsova O.P., Baruseeva N.In., Belkova E.S., Kharchenko N.In. On the improvement of cancer services and improving cancer care to the population of the Russian Federation. // Medical portal «Medical practice». 2009. - P. 11-20.

11.JemalA., Center M.M., DeSantis C., Ward E.M. Global patterns of cancer incidence and mortality rates and trends. // Cancer Epidemiol Biomarkers Prev. 2010. - Vol. 19(8). - P. 1893-1907.

12. Popat K., McQueen K., Feeley T.W. The global burden of cancer. // Best Pract Res Clin Anaesthesiol. 2013. Vol. 27(4). - P. 399-408.

13. World Development Indicators TWB. World Development Indicators: population dynamics. 2015. [Электронный ресурc] - URL: http://wdi.worldbank.org/table/2.1* (Дата обращения: 1 марта 2017).

14. World Health Organization Statistical Information System. 2014. [Электронный pecypc] - URL: http:// www.who.int/healthinfo/statistics/mortality_rawdata/en/index.html (Дата обращения: 1 марта 2017).

15. Mathers C.D., Fat D.M., Inoue M., Rao C., LopezA.D. Counting the dead and what they died from: an assessment of the global status of cause of death data. // Bull World Health Organ. 2005. - Vol. 83(3). - P. 171-177.

16. International classification of disease and related health problem, Tenth Revision, Clinical Modification (ICD-10-CM). 1992. [Электронный ресурc] - URL: http://www.cdc.gov/nchs/icd/icd10cm.htm (Дата обращения: 1 марта 2017).

17.Doll R., Waterhouse J., Muir C.S., Shanmugaratnam K., Powell J. Cancer incidence in five continents. Comparison between registries: age-standardized rates. // IARC Sci Publ. 1982. - Vol. IV(42). - P. 671-675.

18. Bosetti C., Bertuccio P., Malvezzi M., et al. Cancer mortality in Europe, 2005-2009, and an overview of trends since 1980. // Ann Oncol. 2013. - Vol. 24(10). - P. 2657-2671.

19. Chatenoud L., Bertuccio P., Bosetti C., et al. Trends in mortality from major cancers in the Americas: 1980-2010. // Ann Oncol. 2014. - Vol. 25(9). - P. 1843-1853.

20. Parkin D.M., Bray F., Ferlay J., Pisani P. Global cancer statistics, 2002. // CA Cancer J Clin. 2005. - Vol. 55(2). - P. 74-108.

21. Ferlay J., Soerjomataram I., Dikshit R., et al. Cancer incidence and mortality worldwide: sources, methods and major patterns in GLOBOCAN 2012. // Int J Cancer. 2015. - Vol. 136(5). - P. E359-E386.

22. Parkin D.M. The global health burden of infection-associated cancers in the year 2002. // Int J Cancer. 2006. - Vol. 118(12). - P. 3030-3044.

23. Peleteiro B., La Vecchia C., Lunet $N$. The role of Helicobacter pylori infection in the web of gastric cancer causation. // Eur J Cancer Prev. 2012. - Vol. 21(2). - P. 118-125.

24. Bray F., Ren J.S., Masuyer E., Ferlay J. Global estimates of cancer prevalence for 27 sites in the adult population in 2008. // Int J Cancer. 2013. - Vol. 132. - P. 1133-1145.

25. Ferlay J., Shin H.R., Bray F., Forman D., Mathers C., Parkin D.M. Estimates of worldwide burden of cancer in 2008: GLOBOCAN 2008. // Int J Cancer. 2010. - Vol. 127. - P. 2893-2917. 
26. Pourfarzi F., Whelan A., KaldorJ., Malekzadeh R. The role of diet and other environmental factors in the causation of gastric cancer in Iran. - A population based study. // Int J Cancer. 2009. - Vol. 125. - P. 1953-1960.

27. Sjoquist K.M., Burmeister B.H., Smithers B.M., ZalcbergJ.R., Simes R.J., Barbour A., et al. Survival after neoadjuvant chemotherapy or chemoradiotherapy for resectable esophageal carcinoma: an updated meta-analysis. // Lancet Oncol. 2011. - Vol. 12. - P. 681-692.

28. Crew K.D., Neugut A.I. Epidemiology of gastric cancer. // World J Gastroenterol. 2006. - Vol. 12. - P. 354-362.

29. Ferlay J., Shin H.R., Bray F., Forman D., Mathers C., Parkin D.M. Estimates of worldwide burden of cancer in 2008 : GLOBOCAN 2008. // Int J Cancer. 2010. - Vol. 127. - P. 2893-2917.

30. Forman D., Burley V. Gastric cancer: global pattern of the disease and an overview of environmental risk factors. // Best Pract Res Clin Gastroenterol. 2006. - Vol. 20. - P. 633-649.

31. Siegel R., Ma J., Zou Z., Jemal A. Cancer statistics, 2014. // CA Cancer J Clin. 2014. - Vol. 64. - P. 9-29.

32. Arnold M., Moore S.P., Hassler S., Ellison-Loschmann L., Forman D., Bray F. The burden of stomach cancer in indigenous populations: a systematic review and global assessment. // Gut. 2014. - Vol. 63. - P. 64-71.

33. Parsonnet J., Hansen S., Rodriguez L., et al. Helicobacter pylori infection and gastric lymphoma. // $\mathrm{N}$ Engl J Med. 1994. - Vol. 330(18). - P. 1267-1271.

34. Ando T., Goto Y., Maeda O., et al. Causal role of Helicobacter pylori infection in gastric cancer. // World J Gastroenterol. 2006. - Vol. 12(2). - P. 181-186.

35. Aromaa A., Kosunen T.U., Knekt P., et al.: Circulating anti-Helicobacter pylori immunoglobulin A antibodies and low serum pepsinogen I level are associated with increased risk of gastric cancer. // Am J Epidemiol. 1996. Vol. 144(2). - P. 142-149.

36. IARC Working Group on the Evaluation of Carcinogenic Risks to Humans: A review of human carcinogens Part B: biological agents. Vol. 100. // Lyon, France: IARC Press. 2011.

37. Bouvard V., Baan R., StraifK., et al. A review of human carcinogens - Part B: biological agents. // Lancet Oncol. 2009. - Vol. 10 (4). - P. 321-322.

38. Hansson L.E., Nyrén O., Hsing A.W., et al. The risk of stomach cancer in patients with gastric or duodenal ulcer disease. // N Engl J Med. 1996. - Vol. 335(4). - P. 242-249.

39. Althuis M.D., Dozier J.M, Anderson W.F., Devesa S.S., Brinton L.A. Global trends in breast cancer incidence and mortality 1973-1997. // Int J Epidemiol. 2005. - Vol. 34(2). - P. 405-412.

40. Pimenta J.M., Galindo C., Jenkins D., Taylor S.M. Estimate of the global burden of cervical adenocarcinoma and potential impact of prophylactic human papillomavirus vaccination. // BMC Cancer. 2013. - Vol. 13. - P. 553.

41.Jemal A., Bray F., Center M.M., et al. Global cancer statistics. // CA Cancer J Clin. 2011. - Vol. 61. - P. 69-90.

42. Steward B.W., Wild C.P., editors. World Cancer Report 2014. Lyon, France: International Agency for Research on Cancer; 2014.

43. National Cancer Registry Ireland. [Электронный ресурc] - URL: http://www.ncri.ie. (Дата обращения: 21 июля 2014). 44. National Cancer Institute: SEER Stat Fact Sheets: Stomach Cancer. Bethesda, MD: National Cancer Institute, 2015. Available online. Дата обращения: 31 марта, 2016.

45. Ming S.C., Goldman H. Gastric polyps: a histogenetic classification and its relation to carcinoma. // Cancer. 1965. - Vol. 18. - P. 721-726.

46. Utsunomiya J., Maki T., Iwama T., et al. Gastric lesion of familial polyposis coli. // Cancer. 1974. - Vol. 34(3). P. $745-754$.

47. Aarnio M., Salovaara R., Aaltonen L.A., et al. Features of gastric cancer in hereditary non-polyposis colorectal cancer syndrome. // Int J Cancer. 1997. - Vol. 74(5). - P. 551-555.

48. Kurtz R.C., Sherlock P. The diagnosis of gastric cancer. // Semin Oncol. 1985. - Vol. 12(1). - P. 11-18.

49. Boeing H. Epidemiological research in stomach cancer: progress over the last ten years. // J Cancer Res Clin Oncol. 1991. - Vol. 117(2). - P. 133-143.

50. Crew K.D., Neugut A.I. Epidemiology of gastric cancer. // World J Gastroenterol. 2006. - Vol. 12(3). - P. 354-362.

51. Leung W.K., Wu M.S., Kakugawa Y., et al. Screening for gastric cancer in Asia: current evidence and practice. // Lancet Oncol. 2008. - Vol. 9(3). - P. 279-287.

52. Doll R., Peto R. The causes of cancer. // New York, NY: Oxford Press. 1981.

53. World Cancer Research Fund and American Institute for Cancer Research. Policy and action for cancer prevention. // Washington, DC: World Cancer Research Fund and American Institute for Cancer Research. 2009.

54. American Cancer Society. Cancer facts and figures 2015. // Atlanta, GA: American Cancer Society. 2015.

55. U.S. Department of Health and Human Services. Smoking and health report of the advisory committee to the surgeon general of the public health service. // Washington, DC: Public Health Service. 1964.

56. U.S. Department of Health and Human Services. The health consequences of smoking - 50 years of progress. A report from the Surgeon General. // Atlanta, GA: Department of Health and Human Services. Center for Disease Control and Prevention, National Center for Chronic Disease Prevention and Health Promotion. 2014.

57. Shanks T.G., Burns D.M. Disease consequences of cigar smoking. National Cancer Institute, smoking and tobacco control, Monograph 9: Cigars-Health effects and trends. // Washington, DC: National Institutes of Health. 1998.

58. Reynolds P. Smoking and breast cancer. // J Mammary Gland Biol Neoplasia. 2013. - Vol. 18. - P. 15-23.

59. Gaudet M.M., Gapstur S.M., Sun J., Diver W.R., Hannan L.M., Thun M.J. Active smoking and breast cancer risk: original cohort data and meta-analysis. // J Natl Cancer Inst. 2013. - Vol. 105. - P. 515-525. 
60. Centers for Disease Control and Prevention. Vital Signs: nonsmokers' exposure to secondhand smoke-United States, 1999-2008. // MMWR Morb Mortal Wkly Rep. 2010. - Vol. 59. - P. 1141-1146.

61. Norat T., Chan D., Lau R., Vieira R. The associations between food, nutrition and physical activity and the risk of breast cancer. WCRF/AICR systematic literature review continuous updated project report. // London, UK: World Cancer Research Fund / American Institute for Cancer Research. 2008.

62. World Cancer Research Fund/American Institute for Cancer Research. The associations between food, nutrition and physical activity and the prevention of cancer: a global perspective. Washington, DC: World Cancer Research Fund / American Institute for Cancer Research. 2007.

63. Aune D., Greenwood D.C., Chan D.S., Vieira R., Vieira A.R., Navarro Rosenblatt D.A., et al. Body mass index, abdominal fatness and pancreatic cancer risk: a systematic review and non-linear dose-response meta-analysis of prospective studies. // Ann Oncol. 2012. - Vol. 23. - P. 843-852.

64. Kushi L.H., Doyle C., McCullough M., Gansler T., Courneya K., Rock C.L., et al. American Cancer Society guidelines on nutrition and physical activity for cancer prevention: reducing the risk of cancer with healthy food choices and physical activity. // CA Cancer J Clin. 2012. - Vol. 62. - P. 30-67.

65. Calle E.E., Rodriguez C., Walker-Thurmond K., Thun M.J. Overweight, obesity, and mortality from cancer in a prospectively studied cohort of U.S. adults. // N Engl J Med. 2003. - Vol. 348. - P. 1625-1638.

66. Renehan A.G., Tyson M., Egger M., Heller R.F., Zwablen M. Body-mass index and incidence of cancer: a systematic review and meta-analysis of prospective observational studies. // Lancet. 2008. - Vol. 371. - P. 569-578.

67. World Cancer Research Fund and American Institute for Cancer Research. Continuous update project report. Food, nutrition, physical activity, and prostate cancer. // London, UK: World Cancer Research Fund and American Institute for Cancer Research. 2014.

68. Harvie M., Howell A., Vierkant R.A., Kumar N., Cerban J.R., Kelemen L.E., et al. Association of gain and loss of weight before and after menopause with risk of postmenopausal breast cancer in the Iowa women's health study. // Cancer Epidemiol Biomarkers Prev. 2005. - Vol. 14. - P. 656-661.

69. Eliassen A.H., Colditz G.A., Rosner B., Willett W.C., Hankinson S.E. Adult weight change and risk of postmenopausal breast cancer. // JAMA. 2006. - Vol. 296. - P. 193-201.

70. Teras L.R., Goodman M., Patel A.V., Diver W.R., Flanders W.D., Feigelson H.S. Weight loss and postmenopausal breast cancer in a prospective cohort of overweight and obese US women. // Cancer Causes Control. 2011. - Vol. 22. - P. 573-579.

71. World Cancer Research Fund and American Institute for Cancer Research. Food, nutrition, physical activity and the prevention of cancer: a global perspective. // Washington, DC: World Cancer Research Fund and American Institute for Cancer Research. 2007.

72. World Cancer Research Fund and American Institute for Cancer Research. Diet and cancer report. // Washington DC: Research Fund and American Institute for Cancer Research. 2007.

73. Jung S., Spiegelman D., Baglietto L., Bernstein L., Boggs D.A., van den Brandt P.A., et al. Fruit and vegetable intake and risk of breast cancer by hormone receptor status. // J Natl Cancer Inst. 2013. - Vol. 105. - P. 219-236.

74. World Cancer Research Fund and American Institute for Cancer Research. Continuous update project report. Food, nutrition, physical activity, and the prevention of colorectal cancer. // London, UK: Imperial College. 2011.

75. World Cancer Research Fund and American Institute for Cancer Research. Continuous update project report. Food, nutrition, physical activity, and the prevention of pancreatic cancer. // London, UK: Imperial College. 2012.

76. Narod S.A. Alcohol and risk of breast cancer. // JAMA 2011. - Vol. 306. - P. 1920-1921.

77. Mehrabian A.A., Esna-Ashari F., Zham H., Hadizadeb M., Boblooli M., Khayamzadeb M., et al. Gastric cancer prevalence, according to survival data in Iran (National Study - 2007) // Iranian J Pub Health. 2010. - Vol. 39. - P. 27-31.

78. Delpisheh A., Veisani Y., Sayehmiri K., Rahimi E. Esophageal carcinoma: long-term survival in consecutive series of patients through a retrospective cohort study. // Gastroenterol Hepatol Bed Bench. 2014. - Vol. 7. - P. 101-107.

79. Yarbusseini A., Sharifzadeh L., Delpisheh A., Veisani Y., Sayehmiri F., Sayebmiri K. Survival rate of esophageal carcinoma in Iran - a systematic review and meta-analysis. // Iran J Cancer Prevent. 2014. - Vol. 7. - P. 61-65.

80. Mousavi S.M., Somi M.H. Gastric cancer in Iran 1966-2006. // Asian Pac J Cancer Prev. 2009. - Vol. 10. - P. 407-412.

81.Delpisheh A., Veisani Y., Sayehmiri K., Rabimi E. Smoking and Histological Factors Influencing Long-term Survival of Gastric Carcinoma in Consecutive Patient Series. // Middle East J Cancer. 2014. - Vol. 5. - P. 129-135.

82. Leung W.K., Wu M.S., Kakugawa Y., et al. Screening for gastric cancer in Asia: current evidence and practice. // Lancet Oncol. 2008. - Vol. 9(3). - P. 279-287.

83. Hamashima C., Shibuya D., Yamazaki H., et al. The Japanese guidelines for gastric cancer screening. // Jpn J Clin Oncol. 2008. - Vol. 38(4). - P. 259-267.

84. Ohata H., Oka M., Yanaoka K., et al. Gastric cancer screening of a high-risk population in Japan using serum pepsinogen and barium digital radiography. // Cancer Sci. 2005. - Vol. 96(10). - P. 713-720.

85. La Vecchia C., Rota M., Malvezzi M., Negri E. Potential for improvement in cancer management: reducing mortality in the European Union. // Oncologist. 2015. - Vol. 20(5). - P. 495-498.

86. Riecken B., Pfeiffer R., Ma J.L., et al. No impact of repeated endoscopic screens on gastric cancer mortality in a prospectively followed Chinese population at high risk. // Prev Med. 2002. - Vol. 34(1). - P. 22-28.

87. Smith R.A., Manassaram-Baptiste D., Brooks D., Doroshenk M., Fedewa S., Saslow D., et al. Cancer screening in the United States, 2015: a review of current American Cancer Society guidelines and current issues in cancer screening. // CA Cancer J Clin. 2015. - Vol. 65. - P. 30-54. 


\section{References}

1.Jemal A., Bray F., Center M.M., Ferlay J., Ward E., Forman D. Global cancer statistics. CA Cancer J Clin. 2011; 61: 69-90. doi: 10.3322/caac.20107. Epub 2011 Feb 4.

2. Pourboseingholi M.A., Vahedi M., Baghestani A.R. Burden of gastrointestinal cancer in Asia; an overview. Gastroenterol Hepatol Bed Bench. 2015; 8: 19-27.

3. Axel E.M., Dvoirin V.V. Statistics of malignant neoplasms (incidence, mortality, trends, socio-economic damage, life expectancy). Moscow: VONTS AMN SSSR. 1992; 308.

4. Hoffman George. Cancer caused by exposure to low doses: an independent analysis of the problems/transl.from English. Socio-ecological Union. 1994.

5. Yagudina R.I., Kulikov A.Yu.,Arinina E.E. Pharmacoeconomics in Oncology. «Shiko». 2011; 10.

6.Abmad O.B., Boschi-Pinto C., LopezA.D., et al. Age Standardization of Rates: A new WHO Standard. Geneva: World Health Organization. 2001.

7. Jong-wook L. Global health improvement and WHO: shaping the future. Lancet. 2003; 362(9401): 2083-2088. doi: 10.1016/S0140-6736(03)15107-0.

8. Haghighi M.H., Dehghani M., Teshizi S.H., Mahmoodi H. Impact of documentation errors on accuracy of cause of death coding in an educational hospital in Southern Iran. HIM J. 2014; 43(2): 35-42.

9. Silva J.A., Yamaki V.N., Oliveira J.P., et al. Commitment in the completion of the medical death certification. Evaluation in Belem, Para, Brazil in 2010. Rev Assoc Med Bras. 2013; 59(4):335-340. doi: 10.1016/j.ramb.2013.03.001. Epub 2013 Jul 10.

10. Chissov V.I., Starinskiy V.V., Aleksandrova L.M., Lutkovskiy A.S., Savinov V.A., Petrova G.V., Gretsova O.P., Baruseeva N.In., Belkova E.S., Kharchenko N.In. On the improvement of cancer services and improving cancer care to the population of the Russian Federation. Medical portal «Medical practice». 2009.

11.JemalA., Center M.M., DeSantis C., Ward E.M. Global patterns of cancer incidence and mortality rates and trends. Cancer Epidemiol Biomarkers Prev. 2010; 19(8): 1893-1907.

12.Popat K., McQueen K., Feeley T.W. The global burden of cancer. Best Pract Res Clin Anaesthesiol. 2013; 27(4): 399-408.

13. World Development Indicators TWB. World Development Indicators: population dynamics. 2015. Available at: http://wdi.worldbank.org/table/2.1\# (Accessed at 1st March 2017).

14. World Health Organization Statistical Information System. 2014. Available at: http://www.who.int/healthinfo/ statistics/mortality_rawdata/en/index.html (Accessed at 1st March 2017).

15. Mathers C.D., Fat D.M., Inoue M., Rao C., Lopez A.D. Counting the dead and what they died from: an assessment of the global status of cause of death data. Bull World Health Organ. 2005; 83(3): 171-177. doi: /S004296862005000300009 .

16. International classification of disease and related health problem, Tenth Revision, Clinical Modification (ICD10-CM). 1992. Available at: http://www.cdc.gov/nchs/icd/icd10 cm.htm (Accessed at 1st March 2017).

17.Doll R., Waterhouse J., Muir C.S., Shanmugaratnam K., Powell J. Cancer incidence in five continents. Comparison between registries: age-standardized rates. // IARC Sci Publ. 1982; IV(42): 671-675.

18. Bosetti C., Bertuccio P., Malvezzi M., et al. Cancer mortality in Europe, 2005-2009, and an overview of trends since 1980. Ann Oncol. 2013; 24(10): 2657-2671.

19. Chatenoud L., Bertuccio P., Bosetti C., et al. Trends in mortality from major cancers in the Americas: 1980-2010. Ann Oncol. 2014; 25(9): 1843-1853.

20. Parkin D.M., Bray F., Ferlay J., Pisani P. Global cancer statistics, 2002. CA Cancer J Clin. 2005; 55(2): 74-108.

21. Ferlay J., Soerjomataram I., Dikshit R., et al. Cancer incidence and mortality worldwide: sources, methods and major patterns in GLOBOCAN 2012. Int J Cancer. 2015; 136(5): E359-E386. doi: 10.1002/ijc.29210. Epub 2014 Oct 9.

22. Parkin D.M. The global health burden of infection-associated cancers in the year 2002. Int J Cancer. 2006; 118(12): 3030-3044.

23. Peleteiro B., La Vecchia C., Lunet $N$. The role of Helicobacter pylori infection in the web of gastric cancer causation. Eur J Cancer Prev. 2012; 21(2): 118-125.

24. Bray F., Ren J.S., Masuyer E., Ferlay J. Global estimates of cancer prevalence for 27 sites in the adult population in 2008. Int J Cancer. 2013; 132: 1133-1145.

25. Ferlay J., Shin H.R., Bray F., Forman D., Mathers C., Parkin D.M. Estimates of worldwide burden of cancer in 2008: GLOBOCAN 2008. Int J Cancer. 2010; 127: 2893-2917.

26. Pourfarzi F., Whelan A., KaldorJ., Malekzadeh R. The role of diet and other environmental factors in the causation of gastric cancer in Iran - A population based study. Int J Cancer. 2009; 125: 1953-1960. doi: 10.1002/ijc.24499.

27. Sjoquist K.M., Burmeister B.H., Smithers B.M., ZalcbergJ.R., Simes R.J., BarbourA., et al. Survival after neoadjuvant chemotherapy or chemoradiotherapy for resectable esophageal carcinoma: an updated meta-analysis. Lancet Oncol. 2011; 12: 681-692.

28. Crew K.D., Neugut A.I. Epidemiology of gastric cancer. World J Gastroenterol. 2006; 12: 354-362.

29. Ferlay J., Shin H.R., Bray F., Forman D., Mathers C., Parkin D.M. Estimates of worldwide burden of cancer in 2008 : GLOBOCAN 2008. Int J Cancer. 2010; 127: 2893-2917.

30. Forman D., Burley V. Gastric cancer: global pattern of the disease and an overview of environmental risk factors. Best Pract Res Clin Gastroenterol. 2006; 20: 633-649. doi: 10.1016/j.bpg.2006.04.008 
31. Siegel R., Ma J., Zou Z., Jemal A. Cancer statistics, 2014. CA Cancer J Clin. 2014; 64: 9-29.

32. Arnold M., Moore S.P., Hassler S., Ellison-Loschmann L., Forman D., Bray F. The burden of stomach cancer in indigenous populations: a systematic review and global assessment. Gut. 2014; 63: 64-71.

33. Parsonnet J., Hansen S., Rodriguez L., et al. Helicobacter pylori infection and gastric lymphoma. N Engl J Med. 1994; 330 (18): 1267-1271.

34. Ando T., Goto Y., Maeda O., et al. Causal role of Helicobacter pylori infection in gastric cancer. World J Gastroenterol. 2006; 12 (2): 181-186.

35. Aromaa A., Kosunen T.U., Knekt P., et al.: Circulating anti-Helicobacter pylori immunoglobulin A antibodies and low serum pepsinogen I level are associated with increased risk of gastric cancer. Am J Epidemiol. 1996; 144 (2): 142-149.

36. IARC Working Group on the Evaluation of Carcinogenic Risks to Humans: A review of human carcinogens--Part B: biological agents. Vol. 100. Lyon, France: IARC Press. 2011.

37. Bouvard V., Baan R., Straif K., et al. A review of human carcinogens--Part B: biological agents. Lancet Oncol. 2009; 10 (4): 321-322.

38. Hansson L.E., Nyrén O., Hsing A.W., et al. The risk of stomach cancer in patients with gastric or duodenal ulcer disease. N Engl J Med. 1996; 335 (4): 242-249.

39. Althuis M.D., Dozier J.M, Anderson W.F., Devesa S.S., Brinton L.A. Global trends in breast cancer incidence and mortality 1973-1997. Int J Epidemiol. 2005; 34(2): 405-412.

40. Pimenta J.M., Galindo C., Jenkins D., Taylor S.M. Estimate of the global burden of cervical adenocarcinoma and potential impact of prophylactic human papillomavirus vaccination. BMC Cancer. 2013; 13: 553.

41.Jemal A., Bray F., Center M.M., et al. Global cancer statistics. CA Cancer J Clin. 2011; 61: 69-90.

42. Steward B.W., Wild C.P., editors. World Cancer Report 2014. Lyon, France: International Agency for Research on Cancer; 2014.

43. National Cancer Registry Ireland. Available at: http://www.ncri.ie. Accessed July 21, 2014.

44. National Cancer Institute: SEER Stat Fact Sheets: Stomach Cancer. Bethesda, MD: National Cancer Institute, 2015. Available online. Accessed at 31st March, 2016.

45. Ming S.C., Goldman H. Gastric polyps: a histogenetic classification and its relation to carcinoma. Cancer. 1965; 18: 721-726.

46. Utsunomiya J., Maki T., Iwama T., et al. Gastric lesion of familial polyposis coli. Cancer. 1974; 34 (3): 745-754.

47. Aarnio M., Salovaara R., Aaltonen L.A., et al. Features of gastric cancer in hereditary non-polyposis colorectal cancer syndrome. Int J Cancer. 1997; 74 (5): 551-555.

48. Kurtz R.C., Sherlock P. The diagnosis of gastric cancer. Semin Oncol. 1985; 12 (1): 11-18.

49. Boeing $H$. Epidemiological research in stomach cancer: progress over the last ten years. J Cancer Res Clin Oncol. 1991; 117 (2): 133-143.

50. Crew K.D., Neugut A.I. Epidemiology of gastric cancer. World J Gastroenterol. 2006; 12 (3): 354-362.

51. Leung W.K., Wu M.S., Kakugawa Y., et al. Screening for gastric cancer in Asia: current evidence and practice. Lancet Oncol. 2008; 9 (3): 279-287. doi: 10.1016/S1470-2045(08)70072-X.

52. Doll R., Peto R. The causes of cancer. New York, NY: Oxford Press. 1981.

53. World Cancer Research Fund and American Institute for Cancer Research. Policy and action for cancer prevention. Washington, DC: World Cancer Research Fund and American Institute for Cancer Research. 2009.

54. American Cancer Society. Cancer facts and figures 2015. Atlanta, GA: American Cancer Society. 2015.

55. U.S. Department of Health and Human Services. Smoking and health report of the advisory committee to the surgeon general of the public health service. Washington, DC: Public Health Service. 1964.

56. U.S. Department of Health and Human Services. The health consequences of smoking - 50 years of progress. A report from the Surgeon General. Atlanta, GA: Department of Health and Human Services. Center for Disease Control and Prevention, National Center for Chronic Disease Prevention and Health Promotion. 2014.

57. Shanks T.G., Burns D.M. Disease consequences of cigar smoking. National Cancer Institute, smoking and tobacco control, Monograph 9: Cigars-Health effects and trends. Washington, DC: National Institutes of Health. 1998.

58. Reynolds P. Smoking and breast cancer. J Mammary Gland Biol Neoplasia. 2013; 18: 15-23.

59. Gaudet M.M., Gapstur S.M., Sun J., Diver W.R., Hannan L.M., Thun M.J. Active smoking and breast cancer risk: original cohort data and meta-analysis. J Natl Cancer Inst. 2013; 105: 515-525. doi: 10.1093/jnci/djt023. Epub 2013 Feb 28.

60. Centers for Disease Control and Prevention. Vital Signs: nonsmokers' exposure to secondhand smoke - United States, 1999-2008. MMWR Morb Mortal Wkly Rep. 2010; 59: 1141-1146.

61. Norat T., Chan D., Lau R., Vieira R. The associations between food, nutrition and physical activity and the risk of breast cancer. WCRF / AICR systematic literature review continuous updated project report. London, UK: World Cancer Research Fund / American Institute for Cancer Research. 2008.

62. World Cancer Research Fund/American Institute for Cancer Research. The associations between food, nutrition and physical activity and the prevention of cancer: a global perspective. Washington, DC: World Cancer Research Fund/American Institute for Cancer Research. 2007.

63. Aune D., Greenwood D.C., Chan D.S., Vieira R., Vieira A.R., Navarro Rosenblatt D.A., et al. Body mass index, abdominal fatness and pancreatic cancer risk: a systematic review and non-linear dose-response meta-analysis of prospective studies. Ann Oncol. 2012; 23: 843-852. 
64. Kushi L.H., Doyle C., McCullough M., Gansler T., Courneya K., Rock C.L., et al. American Cancer Society guidelines on nutrition and physical activity for cancer prevention: reducing the risk of cancer with healthy food choices and physical activity. CA Cancer J Clin. 2012; 62: 30-67. doi: 10.3322/caac.20140.

65. Calle E.E., Rodriguez C., Walker-Thurmond K., Thun M.J. Overweight, obesity, and mortality from cancer in a prospectively studied cohort of U.S. adults. N Engl J Med. 2003; 348: 1625-1638.

66. Renehan A.G., Tyson M., Egger M., Heller R.F., Zwablen M. Body-mass index and incidence of cancer: a systematic review and meta-analysis of prospective observational studies. Lancet. 2008; 371: 569-578.

67. World Cancer Research Fund and American Institute for Cancer Research. Continuous update project report. Food, nutrition, physical activity, and prostate cancer. London, UK: World Cancer Research Fund and American Institute for Cancer Research. 2014.

68. Harvie M., Howell A., Vierkant R.A., Kumar N., Cerban J.R., Kelemen L.E., et al. Association of gain and loss of weight before and after menopause with risk of postmenopausal breast cancer in the Iowa women's health study. Cancer Epidemiol Biomarkers Prev. 2005; 14: 656-661. doi: 10.1158/1055-9965.EPI-04-0001.

69. Eliassen A.H., Colditz G.A., Rosner B., Willett W.C., Hankinson S.E. Adult weight change and risk of postmenopausal breast cancer. JAMA. 2006; 296: 193-201.

70. Teras L.R., Goodman M., Patel A.V., Diver W.R., Flanders W.D., Feigelson H.S. Weight loss and postmenopausal breast cancer in a prospective cohort of overweight and obese US women. Cancer Causes Control. 2011; 22: 573-579.

71. World Cancer Research Fund and American Institute for Cancer Research. Food, nutrition, physical activity and the prevention of cancer: a global perspective. Washington, DC: World Cancer Research Fund and American Institute for Cancer Research. 2007.

72. World Cancer Research Fund and American Institute for Cancer Research. Diet and cancer report. Washington DC: Research Fund and American Institute for Cancer Research. 2007.

73.Jung S., Spiegelman D., Baglietto L., Bernstein L., Boggs D.A., van den Brandt P.A., et al. Fruit and vegetable intake and risk of breast cancer by hormone receptor status. J Natl Cancer Inst. 2013; 105: 219-236. doi: 10.1093/jnci/djs635. Epub 2013 Jan 24.

74. World Cancer Research Fund and American Institute for Cancer Research. Continuous update project report. Food, nutrition, physical activity, and the prevention of colorectal cancer. London, UK: Imperial College. 2011.

75. World Cancer Research Fund and American Institute for Cancer Research. Continuous update project report. Food, nutrition, physical activity, and the prevention of pancreatic cancer. London, UK: Imperial College. 2012.

76. Narod S.A. Alcohol and risk of breast cancer. JAMA 2011; 306: 1920-1921.

77. Mehrabian A.A., Esna-Ashari F., Zham H., Hadizadeb M., Boblooli M., Khayamzadeb M., et al. Gastric cancer prevalence, according to survival data in Iran (National Study-2007). Iranian J Pub Health. 2010; 39: 27-31.

78. Delpisheh A., Veisani Y., Sayehmiri K., Rabimi E. Esophageal carcinoma: long-term survival in consecutive series of patients through a retrospective cohort study. Gastroenterol Hepatol Bed Bench. 2014; 7: 101-107.

79. Yarhusseini A., Sharifzadeb L., Delpisheb A., Veisani Y., Sayebmiri F., Sayehmiri K. Survival rate of esophageal carcinoma in Iran - a systematic review and meta-analysis. Iran J Cancer Prevent. 2014; 7: 61-65.

80. Mousavi S.M., Somi M.H. Gastric cancer in Iran 1966-2006. Asian Pac J Cancer Prev. 2009; 10: 407-412.

81.Delpisheb A., Veisani Y., Sayebmiri K., Rabimi E. Smoking and Histological Factors Influencing Long-term Survival of Gastric Carcinoma in Consecutive Patient Series. Middle East J Cancer. 2014; 5: 129-135.

82. Leung W.K., Wu M.S., Kakugawa Y., et al. Screening for gastric cancer in Asia: current evidence and practice. Lancet Oncol. 2008; 9 (3): 279-287.

83. Hamashima C., Shibuya D., Yamazaki H., et al. The Japanese guidelines for gastric cancer screening. Jpn J Clin Oncol. 2008; 38 (4): 259-267. doi: 10.1093/jjco/hyn017. Epub 2008 Mar 14.

84. Obata H., Oka M., Yanaoka K., et al. Gastric cancer screening of a high-risk population in Japan using serum pepsinogen and barium digital radiography. Cancer Sci. 2005; 96 (10): 713-720.

85. La Vecchia C., Rota M., Malvezzi M., Negri E. Potential for improvement in cancer management: reducing mortality in the European Union. Oncologist. 2015; 20(5): 495-498. doi: 10.1634/theoncologist.2015-0011. Epub 2015 Apr 17. 86. Riecken B., Pfeiffer R., Ma J.L., et al. No impact of repeated endoscopic screens on gastric cancer mortality in a prospectively followed Chinese population at high risk. Prev Med. 2002; 34 (1): 22-28.

87. Smith R.A., Manassaram-Baptiste D., Brooks D., Doroshenk M., Fedewa S., Saslow D., et al. Cancer screening in the United States, 2015: a review of current American Cancer Society guidelines and current issues in cancer screening. CA Cancer J Clin. 2015; 65: 30-54. doi: 10.3322/caac.21261. Epub 2015 Jan 8. 